\title{
Competencias del profesor de Ciencia de la Información, Bibliotecología y Archivística en América Latina y España
}

\author{
Competences of the professor of Information Science, Library Science and \\ Archival Science in Latin America and Spain
}

\author{
Orlanda Jaramillo \\ http://orcid.org/0000-0002-3271-0256 \\ Leidy Marisol Salazar Álvarez \\ http://orcid.org/0000-0003-2358-2587 \\ María José Mercado Castrillón \\ http://orcid.org/0000-0002-2336-7425 \\ Universidad de Antioquia, Medellín, Colombia \\ Contacto: orlanda.jaramillo@udea.edu.co
}

\begin{abstract}
Resumen
El texto aborda las competencias del profesor de Ciencia de la Información, Bibliotecología y Archivística en América Latina y España, a partir de un enfoque mixto cuya búsqueda documental revela que han sido poco afrontadas. Hay que puntualizar que el perfil docente debe mirarse, reflexionarse y evaluarse según las competencias y habilidades; ello se traduce en un abordaje de los contenidos curriculares con base en las dimensiones cognitivas (saber), de gestión-aplicación (hacer), actitudinales (ser) y comunicativas (convivir). El trabajo advierte sobre la transversalidad de las tecnologías de la información y comunicación (TIC) en los planes de estudio, para que cumplan un papel preponderante como herramienta en el desarrollo disciplinar. En consecuencia, se propone un listado de competencias para el profesor del área de las TIC según los componentes de la dimensión cognitiva.
\end{abstract}

Palabras clave: perfil docente; profesor universitario; bibliotecología; ciencia de la información; archivística; competencias; TIC; gerencia de información; fundamentación teórica; organización y representación de la información.

\begin{abstract}
The paper addresses the competences of the professor in Information Science, Library Science and Archivology in Latin America and Spain from a mixed approach, where the documentary search shows the little tackled of the subject. Therefore, it points out the teaching profile should be viewed, reflected and evaluated from the competencies and skills; which is translated into the approach of curricular contents from the cognitive (to know), management-application (to do), attitu-
\end{abstract}


dinal (to be) and communicative (to cohexist) dimensions. The work warns of the transversality of ICT in the curricula, where they play an important role as a tool in the disciplinary development. Consequently, a list of competences for the ICT area teacher is proposed from the components of the cognitive dimension.

Keywords: professor profile; university professor; library science; science information; archivology; competencies; ICT; theory foundation; organization and representation of the information

$$
\text { Recibido: 02.08.17 Aceptado: 21.10.17 }
$$

\section{Introducción}

El perfil del profesor universitario en Ciencia de la Información, Bibliotecología y Archivística responde a las funciones misionales de la universidad: docencia, investigación y extensión-proyección, en las que la docencia está relacionada con las competencias de carácter disciplinar y pedagógico; la investigación con las competencias y habilidades para la generación de nuevos conocimientos y la transformación de sus saberes, actitudes y aptitudes en el campo disciplinar; y la extensión-proyección a la sociedad con las competencias para lograr el impacto del ejercicio docente en la solución de los problemas relacionados con la transferencia de la información en la sociedad. Dichas competencias componen el perfil del profesor y están en correspondencia con los objetivos curriculares de formación y con los contenidos, los métodos y las necesidades del contexto. Así, el perfil del profesor debe responder a los procesos de producción, recuperación, organización, tratamiento y difusión de la información y el conocimiento, procesos de carácter transversal debido al uso y manejo de las TIC y la administración-gerencia, en consonancia con los planteamientos que la Special Library Association (2016) expone en el documento de las Core Competencies. En esta línea se planteó la investigación Perfil del docente y su vinculación con el modelo educativo en la educación bibliotecológica y de documentación en Iberoamérica y El Caribe, que tuvo como primera fase la identificación de los estudios realizados acerca del perfil del profesor. Los resultados hacen parte del presente texto.

La identificación de los estudios sobre el perfil empleó el método de búsqueda y análisis documental que se realizó en cuatro grandes áreas: fundamenta- 
ción teórica, organización y representación de la información y el conocimiento, administración-gerencia de la información y tecnologías de la información y la comunicación (TIC). Se estableció que las competencias cognitivas que debe desarrollar el profesor en el área de fundamentación se relacionan con el objeto epistémico de la disciplina, con la generación y aplicación de conocimiento teórico y con los factores de desempeño profesional. Ahora bien, en el área de organización y representación de la información y el conocimiento se exigen competencias de carácter cognitivo, procedimental y actitudinal, determinadas por las tendencias de los contextos sociales, académicos, tecnológicos y laborales, para garantizar la conservación, almacenamiento, acceso, recuperación y uso de la información. En tanto, el perfil del profesor del área de administración-gerencia requiere competencias para el análisis estratégico e innovador de productos y servicios de información, al igual que habilidades y actitudes de liderazgo para gestionar la información como una ventaja competitiva. En el caso del área de las TIC, el profesor debe desarrollar competencias relacionadas con el conocimiento y uso de plataformas educativas, contenidos digitales y aplicaciones para la evaluación y representación del conocimiento. También debe demostrar cualidades de visionario y de profesional abierto a los cambios y al trabajo interdisciplinar. No obstante el desarrollo de competencias en estas áreas, la investigación identificó que no es posible hablar de un único perfil que integre todas las áreas. Si bien se examina el perfil del profesor en las cuatro áreas, se hace énfasis en la propuesta del perfil del profesor del área de las TIC, debido a su carácter de eje transversal a las áreas que conforman el desempeño en las disciplinas de la ciencia de la información.

\section{Método}

El desarrollo investigativo corresponde a un proceso exploratorio con enfoque mixto; el método de investigación y análisis documental fue realizado en el metabuscador Carrot2, en las bases de datos DialNet, DOAJ, EBSCO, Emeral@, Science Direct, Web of Science, en repositorios E-lis, Redalyc, SciELO y en las instituciones que ofrecen programas de formación de profesionales de la información (Universidades de Antioquia, Pontificia Universidad Javeriana, La Salle 
y del Quindío). Los documentos recuperados se categorizaron y analizaron con el software de minería de texto Voyant que permitió la identificación de palabras, frases y términos repetidos en uno o varios textos y la visualización de los datos en nubes de palabras, estadísticas de concurrencia y vínculos conceptuales. En la búsqueda se establecieron criterios de temporalidad (2005-2015) y geográficos (América Latina y España). Estos términos se parametrizaron mediante el uso de operadores boleanos y comillas ("') para construir ecuaciones de búsquedas diversas y garantizar mayores resultados en la exploración. Los términos de búsqueda forman parte del anexo uno.

\section{Resultados}

En una perspectiva cuantitativa se encontraron 40.086 documentos relacionados con el perfil docente en cada una de las cuatro áreas definidas. El uso del metabuscador Carrot2 es la fuente con el mayor número de documentos seleccionados, que permitió la localización de trabajos alojados en diversas fuentes; seguido de las bases de datos de EBSCO, DialNet, Redalyc y Science Direct. Estos resultados se pueden apreciar en la siguiente tabla.

Tabla 1. Resultados por fuente consultada

\begin{tabular}{|c|c|c|c|c|c|c|c|c|}
\hline \multirow{3}{*}{ Fuentes } & \multicolumn{8}{|c|}{ Áreas } \\
\hline & \multicolumn{2}{|c|}{$\begin{array}{c}\text { Fundamentación } \\
\text { teórica }\end{array}$} & \multicolumn{2}{|c|}{$\begin{array}{l}\text { Organización- } \\
\text { representación de la } \\
\text { información }\end{array}$} & \multicolumn{2}{|c|}{$\begin{array}{c}\text { Gerencia- } \\
\text { administración }\end{array}$} & \multicolumn{2}{|c|}{$T I C$} \\
\hline & Recuperado & Seleccionado & Recuperado & Seleccionado & Recuperado & Seleccionado & Recuperado & Seleccionado \\
\hline Carrot2 & 1.607 & 24 & 1.382 & 32 & 4.693 & 19 & 2.708 & 104 \\
\hline DialNet & 2.179 & 41 & 2.534 & 10 & 526 & 3 & 428 & 21 \\
\hline DOAJ & 693 & 3 & 362 & 4 & 58 & 1 & 230 & 9 \\
\hline EBSCO & 1.541 & 19 & 495 & 2 & 2.014 & 0 & 1.215 & 7 \\
\hline E-lis & 146 & 6 & 181 & 11 & 406 & 7 & 330 & 18 \\
\hline Emerald@ & 347 & 12 & 143 & 3 & 1.799 & 0 & 9 & 0 \\
\hline $\begin{array}{c}\text { Pontificia U. } \\
\text { Javeriana }\end{array}$ & 51 & 1 & 51 & 1 & 389 & 0 & 59 & 0 \\
\hline Redalyc & 907 & 39 & 272 & 16 & 3.060 & 5 & 994 & 29 \\
\hline SciELO & 279 & 3 & 638 & 17 & 44 & 1 & 16 & 2 \\
\hline $\begin{array}{l}\text { Science } \\
\text { Direct }\end{array}$ & 812 & 9 & 1.112 & 2 & 2.026 & 0 & 383 & 0 \\
\hline $\begin{array}{c}\text { U. de } \\
\text { Antioquia }\end{array}$ & 130 & 3 & 215 & 3 & 23 & 3 & 434 & 5 \\
\hline $\begin{array}{l}\text { U. de La } \\
\text { Salle }\end{array}$ & 214 & 1 & 330 & 5 & 255 & 0 & 552 & 0 \\
\hline $\begin{array}{l}\text { U. del } \\
\text { Quindio }\end{array}$ & 35 & 0 & 44 & 0 & 0 & 0 & 0 & 0 \\
\hline $\begin{array}{l}\text { Web of } \\
\text { Science }\end{array}$ & 436 & 19 & 194 & 3 & 72 & 0 & 33 & 3 \\
\hline Total & 9.377 & 179 & 7.953 & 109 & 15.365 & 39 & 7.391 & 198 \\
\hline
\end{tabular}

Fuente: Elaboración propia. 
Del total de documentos encontrados se hizo una preselección de 525 estudios, de los cuales se seleccionaron 366 por su pertinencia con el perfil del docente en ciencia de la información, bibliotecología y archivística en América Latina y España y, finalmente, para el proceso de investigación se analizaron 60 documentos. Los datos se exponen a continuación.

Tabla 2. Resultados por áreas

\begin{tabular}{|l|r|r|r|r|r|}
\hline \multirow{2}{*}{ Documentos } & \multicolumn{5}{|c|}{ Áreas } \\
\cline { 2 - 7 } & Fundamentación & Organización & Gerencia & TIC & Total \\
\hline Recuperados & 9.377 & 7.953 & 15.365 & 7.391 & $\mathbf{4 0 . 0 8 6}$ \\
\hline Preseleccionados & 179 & 109 & 39 & 198 & $\mathbf{5 2 5}$ \\
\hline Seleccionados & 131 & 109 & 21 & 105 & $\mathbf{3 6 6}$ \\
\hline Analizados & 25 & 20 & 6 & 9 & $\mathbf{6 0}$ \\
\hline
\end{tabular}

Fuente: Elaboración propia.

En cuanto a la temporalidad, se encontró que 2012 es el año con el porcentaje más alto de publicaciones, seguido de 2009. Con relación al lugar, España y México reportan el mayor número de publicaciones, quizá porque estos países hacen parte del Seminario de Educación Bibliotecológica, del Instituto de Investigaciones Bibliotecológicas y de la Información (IIBI) de la Universidad Autónoma de México (UNAM), donde se trabajan temas de educación bibliotecológica. A continuación se relacionan los documentos por año de publicación y los países de procedencia de los autores que reportan trabajos seleccionados.

Gráfico 1. Documentos por fecha de publicación y países de procedencia

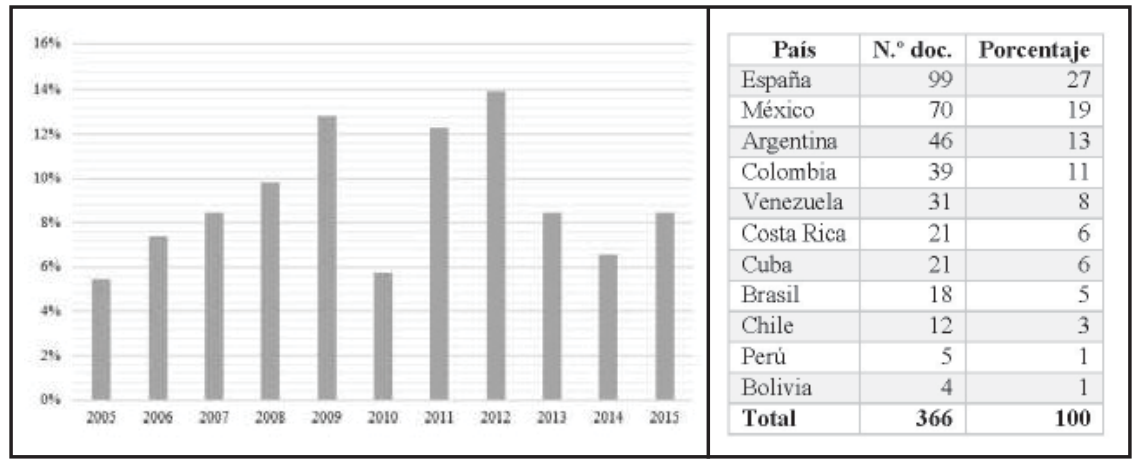

Fuente: Elaboración propia. 
En la perspectiva cualitativa, se consideraron las áreas y competencias determinadas por la Special Library Association (SLA) en el documento Competencies for Information Professionals of the 21 st Century (2016), competencias que se sintetizan en ocho áreas llamadas: fundamentación

teórica, organización de la información y el conocimiento, TIC, recuperación y análisis de la información, recursos de información y del conocimiento, servicios de información y del conocimiento, ética informacional y competencias complementarias. Para efectos de la investigación, estas áreas se reagruparon en cuatro: fundamentación teórica, organización y representación de la información, gerencia-administración de la información y TIC.

Los 60 estudios analizados dan cuenta de las competencias del profesor en Ciencia de la Información, Bibliotecología y Archivística en América Latina y España; de ellos, 25 estudios pertenecen al área de fundamentación teórica. Aquí los autores más representativos son: Setién Quesada, Rendón Rojas, Alfaro López, Ríos Ortega, Linares Columbié, González de Gómez, Ávila Araújo y Parada, quienes se centran en el establecimiento de enfoques epistémicos para el desarrollo de directrices básicas en la enseñanza de la disciplina y las buenas prácticas del quehacer bibliotecológico, al tiempo que las definen como competencias importantes que debe adoptar el profesor mediante la investigación. Los estudios analizados se exponen en el anexo dos.

En cuanto al área de organización y representación de la información, con 20 estudios analizados, el autor más representativo es Rodríguez García, quien además es citado por otros autores en el área; Rodríguez aborda la aplicación de métodos, técnicas e instrumentos para la organización y análisis de la información, apoyados por las TIC como herramientas para garantizar la conservación, almacenamiento, acceso, recuperación y uso de la información. El anexo tres presenta los estudios analizados en el área de organización y tratamiento de la información (OTI).

El área de gerencia-administración de la información presenta una condición particular, debido a que corresponde a saberes propios de las ciencias administra- 
tivas; aquí el profesor no necesariamente requiere formación en ciencia de la información, bibliotecología y archivística, tal como lo demuestran los seis estudios seleccionados. En esta área se destacan dos autores: Flores de la Fuente y Téllez Tolosa, quienes exponen las competencias enfocadas al pensamiento estratégico, habilidades gerenciales y liderazgo; estudios que hacen parte del anexo cuatro.

Por su parte, en el área de las TIC los nueve estudios analizados ponen énfasis en la urgencia de incluir las tecnologías de la información y la comunicación como eje transversal en los planes de estudio, lo que evidencia la relación de estas investigaciones con la formación de profesionales, más no propiamente con el perfil del profesor. Situación diferente presenta el trabajo de Tejada Artigas, quien propone competencias específicas para el profesor de esta área y el de Garduño Vera, quien resalta las competencias del profesor para desarrollar su labor desde la virtualidad (manejo de dispositivos y herramientas tecnológicas, servicios de la web social y alfabetización informacional). Véase el listado de estudios analizados en el anexo cinco.

A partir de estos 60 estudios se desarrolló el acercamiento al perfil del profesor de Ciencia de la Información, Bibliotecología y Archivística en América Latina y España, ya que exponen las competencias mínimas del docente en cada una de las áreas que comprenden dichas disciplinas. En consecuencia, en fundamentación teórica las competencias del profesor están relacionadas con la generación de nuevos conocimientos epistémicos para el impulso constante de la disciplina desde la investigación y el desarrollo de procesos de enseñanza-aprendizaje. En el área de organización y representación de la información se requieren habilidades y destrezas de carácter lógico, matemático, lingüístico, comunicativo, cognitivo y psicológico para el desarrollo de los procesos de análisis de información y conocimiento, además del dominio de los diferentes estándares de catalogación y clasificación. Por otro lado, en el área de administración-gerencia se enfatiza en el crecimiento de habilidades de liderazgo y pensamiento estratégico e innovador. Finalmente, se evidenció un eje trasversal en el perfil de los docentes de estas áreas con competencias en TIC, razón por la cual, a continuación, se hace una propuesta acerca de las competencias específicas en esta área. 


\section{Propuesta de perfil del profesor de TIC}

Las competencias definen, perfeccionan y estructuran el perfil del profesor, pero deben responder tanto a conocimientos específicos del área como a conocimientos pedagógicos, jurídico-normativos e institucionales. En el caso concreto del perfil de profesor del área de las TIC, es necesario que desarrolle competencias para trascender del carácter instrumental a mecanismos de desarrollo social, en aspectos relacionados con procesos pedagógicos, disciplinares y de crecimiento humano que faciliten la apropiación sociocrítica de las tecnologías. Ello se traduce en la acción de integrar las TIC a partir de dos dimensiones: una transversal y otra específica. La primera hace referencia al uso de las TIC desde el pensamiento crítico; la segunda alude al aprovechamiento de las TIC para la generación de procesos de innovación.

Siguiendo la propuesta de competencias tecnológicas de Tejada Artigas (ca. 2011) para el profesor de Bibliotecología y Ciencia de la Información, respecto a las habilidades de la profesión, con el establecimiento de competencias de acuerdo con el modelo educativo — presencial, semipresencial o virtual—, y con competencias según el rol del profesor universitario, en consonancia, además, con los resultados obtenidos de las competencias expuestas por la UNESCO (2008) y del Informe de Tendencias de IFLA (2013), se propone un perfil del profesor del área de TIC fundamentado en cuatro aspectos: jurídico y normativo, institucional (políticas y directrices), pedagógico (procesos de enseñanza-aprendizaje) y específico (uso, aplicación, difusión y generación de conocimientos a partir de las TIC). Estos aspectos y competencias hacen parte de la siguiente tabla. 
Tabla 3. Componentes del profesor del área de TIC

\begin{tabular}{|c|c|}
\hline Aspectos & Competencias \\
\hline $\begin{array}{l}\text { Conocimientos } \\
\text { en el área }\end{array}$ & $\begin{array}{l}\text { Sistemas operativos. } \\
\text { Ofimática. } \\
\text { Lenguajes básicos de programación. } \\
\text { Conceptos sobre arquitectura y sistemas de información. } \\
\text { Plataformas y soportes tecnológicos para la organización y tratamiento de la información. } \\
\text { Herramientas de la web para la organización y tratamiento de la información. } \\
\text { Apps - Aplicaciones móviles. } \\
\text { Internet de las cosas. } \\
\text { Herramientas y contenidos para lectoescritura digital. } \\
\text { Herramientas para la virtualidad y enseñanza a distancia. } \\
\text { Herramientas y contenidos para la visibilidad web y uso de redes sociales y otras } \\
\text { comunidades para difundir sus publicaciones: self-marketing. } \\
\text { Procesos de gestión de contenido web en redes sociales. } \\
\text { La web semántica. } \\
\text { Sociedades hiperconectadas relacionadas con la ciencia de la información. } \\
\text { Beneficios y dificultades que acarrean las TIC en espacios fisicos y virtuales de las unidades } \\
\text { de información. } \\
\text { ALFIN (cuándo, dónde y por qué necesita información, cómo evaluarla, utilizarla y } \\
\text { comunicarla, con su uso ético). } \\
\text { Humanidades digitales. } \\
\text { Herramientas de actualización permanente (RSS). } \\
\text { Estrategias de acceso a la información pública (gobierno en linea y datos abiertos). } \\
\text { Herramientas de análisis, transferencia y almacenamiento de información (bibliometria, } \\
\text { SIGB, mineria de texto, BigData, gestores bibliográficos). } \\
\text { Segundo idioma (lee, escribe y escucha en inglés). }\end{array}$ \\
\hline $\begin{array}{l}\text { Juridico- } \\
\text { normativos }\end{array}$ & $\begin{array}{l}\text { Aspectos juridicos y normativos relacionados con las TIC (propiedad intelectual, derechos } \\
\text { de autor y tipos de licencias, políticas, normas, directrices). } \\
\text { Valor y el costo de la información. } \\
\text { Reconoce aspectos relacionados con las TIC en cuanto a temas de seguridad de datos. }\end{array}$ \\
\hline Pedagógicos & $\begin{array}{l}\text { Plataformas de educación virtual y aprendizaje a distancia. } \\
\text { Herramientas y recursos TIC para el proceso enseñanza-aprendizaje. } \\
\text { Aspectos cognitivos de los estudiantes. } \\
\text { Experiencias educativas en el área. } \\
\text { Métodos y modelos pedagógicos. }\end{array}$ \\
\hline Institucionales & $\begin{array}{l}\text { La institución, los programas y las asignaturas que imparte. } \\
\text { Macro, meso y micro currículo y sus relaciones. }\end{array}$ \\
\hline
\end{tabular}

Fuente: Elaboración propia. 
Estas competencias a su vez deben ser conjugadas desde las dimensiones planteadas por Delors (1996): aprender a aprender, aprender a hacer, aprender a ser y aprender a convivir; que en el caso del perfil del profesor de Ciencia de la Información, Bibliotecología y Archivística se traduce en competencias cognitivas, de gestión-aplicación, actitudinales y comunicativas. Las cognitivas hacen referencia a los conocimientos del área; las de gestión-aplicación corresponden a la aplicación de los conocimientos en escenarios reales; las actitudinales comprenden las cualidades personales; y la comunicativa tiene que ver con las capacidades para la difusión social del conocimiento, la transición de experiencias y construcción de comunidad académica. Finalmente, para la propuesta del perfil del profesor del área de TIC en Ciencia de la Información, Bibliotecología y Archivística, se toman los componentes propios de la dimensión cognitiva, ya que a partir de esta dimensión es posible visualizar las competencias del profesor en el área de TIC; se advierte que las otras tres dimensiones, gestión-aplicación, actitudinal y comunicativa, son posibles desde la existencia de la cognitiva; es decir, se aplica y difunde lo que se sabe, se tiene y se siente.

\section{Conclusiones}

Los resultados encontrados permiten afirmar que el tema del perfil docente en el área de Ciencia de la Información, Bibliotecología y Archivística aún no forma parte de las agendas de investigación, pues si bien hay una cantidad considerable de estudios acerca de su perfil, especialmente en temas de definición y actualización de las competencias profesionales, se evidenció la escasez de trabajos sobre las competencias específicas del profesor universitario adscrito a las áreas core de las disciplinas. En tal sentido, si los planes de estudio deben responder a las exigencias y tendencias disciplinares y de la sociedad, el perfil de los profesores tiene que garantizar el cumplimiento de estas exigencias, por lo que se hace necesario el surgimiento de perfiles polivalentes de los profesores en Ciencia de la Información, Bibliotecología y Archivística.

El estudio sobre el perfil del profesor permite puntualizar que el ejercicio docente se debe mirar, reflexionar y medir a partir de las competencias y habili- 
dades, y no solo mediante un "qué es"; lo que se traduce en el tipo de abordaje requerido para el desarrollo de los contenidos curriculares, según lo que se debe saber hacer, complementado con lo que demanda el medio laboral o contexto ocupacional. Estos elementos permiten que el profesional disponga de competencias y mecanismos de adaptación para aplicar su saber hacer en cualquier entorno.

Con relación a los resultados obtenidos en el proceso de búsqueda, se concluye que las fuentes que arrojaron el mayor número de trabajos pertinentes para la investigación fueron DialNet, Redalyc, Scielo y E-lis. Se destaca la importancia del metabuscador Carrot2, ya que permitió la identificación inicial de varios documentos alojados en las bases de datos previamente mencionadas. Con respecto al lugar de publicación, España es el país que cuenta con el mayor número de estudios en el tema de investigación, seguido de México y Colombia; los tres países, con sus correspondientes autores, forman parte del Seminario de Educación Bibliotecológica del IIBI-UNAM. En esta misma línea, las fechas de publicación tienen correspondencia con la trayectoria del Seminario, con las siguientes precisiones: los trabajos de 2009 se orientan a la construcción del perfil por competencias; los publicados entre 2011-2012 hacen énfasis en la actualización de las competencias, a partir de la incidencia de las TIC, y los de 2015 se centran en la pertinencia del perfil y su correspondencia con las demandas del mercado laboral.

Una característica del profesor del área de TIC es la apertura, disposición y actualización para las nuevas tecnologías, que se manifiesta en la actitud, reconocimiento, posición crítica y evaluadora de las diversas tecnologías y su afectación en los contextos, que contribuye, orienta y muestra caminos para la formación de los profesionales de la información. Esta particularidad obedece a los avances tecnológicos, las nuevas formas de gestión de la información y del conocimiento y las exigencias del mercado, que demandan cambios en el desempeño de estos profesionales; cambios que reclaman, de un lado, la revisión y adaptación de los perfiles docentes responsables de la formación profesional y, de otro, la revisión y actualización de los contenidos de las asignaturas del área de las TIC.

El trabajo evidencia la urgencia de la incorporación de las TIC de manera transversal en los planes de estudio, de tal manera que no sean miradas y defini- 
das como un área separada, sino como un componente de los diferentes procesos inherentes al quehacer de los profesionales de la información: generación, recuperación, organización y tratamiento, evaluación y difusión de la información y el conocimiento. De ahí que sea impostergable en la formación, y con ello en el perfil del profesor, la familiaridad y puesta en práctica las tendencias de las TIC a los procesos formativos: virtualidad, digitalización, dispositivos tecnológicos, herramientas, servicios y gestión de contenido web en redes sociales, soportes tecnológicos para la gestión documental, web semántica, Apps, integración tecnológica (internet de las cosas), uso de equipos modernos como drones y aparatos de realidad virtual. Estas tendencias no solo generan correspondencia con el conocimiento y aplicación de la tecnología blanda-software, sino también con la tecnología dura-hardware.

Se advierte de la transversalidad de las TIC en los planes de estudio, de modo que la formación profesional cada vez guarde mayor concordancia con las demandas sociales y laborales; las TIC deben cumplir un papel preponderante como herramienta en el desarrollo disciplinar. Finalmente, es necesario señalar que las TIC en la Bibliotecología, Archivística y la Ciencia de la Información, constituyen una herramienta inherente al ejercicio docente y al desenvolvimiento del profesional en la sociedad de la información, y como tal debe ser considerada, y no como una disciplina.

\section{Agradecimientos}

Texto derivado de la investigación: Perfil del docente y su vinculación con el modelo educativo en la educación bibliotecológica y de documentación en Iberoamérica y El Caribe. Investigación coordinada por el Instituto de Investigaciones Bibliotecológicas y de la Información de la Universidad Nacional Autónoma de México. 


\section{Referencias bibliográficas}

Delors, J. (1996). La Educación encierra un tesoro. Informe a la UNESCO de la Comisión Internacional sobre la Educación para el siglo XXI. Madris, España: Santillana, Ediciones UNESCO.

IFLA. ([2013]). ¿Surcando las olas o atrapados en la marea? Navegando el entorno en evolución de la información. Percepciones del IFLA Trend Report. http://www.abinia.org/surcando_las_olas_o_atrapados_en_la_marea.pdf [17 de febrero de 2016]

Jaramillo, O. (2017). Perfil del docente y su vinculación con el modelo educativo en la educación bibliotecológica y de documentación en Iberoamérica y El Caribe (Informe de investigación). Medellín, Colombia: Universidad de Antioquia, Escuela Interamericana de Bibliotecología.

Special Libraries Association. (2016). Competencies for Information Professionals. https://www.sla.org/about-sla/competencies/\#sthash.ZfScXdkM. dpuf [20 de octubre de 2016]

Tejada Artigas, C. M. (2011). Competencias tecnológicas de los profesores universitarios en información y documentación. http://iibi.unam.mx/ publicaciones/280/tic\%20educacion\%20bibliotecologica\%20Competencias\%20Tecnologicas\%20Carlos\%20Miguel\%20Tejada\%20Artigas. html

UNESCO. (2008). Estándares de competencias en TIC para docentes. http:// www.oei.es/tic/UNESCOEstandaresDocentes.pdf [28 de junio de 2016] 
Anexo 1: Palabras clave

\begin{tabular}{|c|c|c|}
\hline \multirow[t]{2}{*}{ Área } & \multicolumn{2}{|r|}{ Palabras clave } \\
\hline & Español & Inglés \\
\hline $\begin{array}{l}\text { Fundamentación } \\
\text { teónica }\end{array}$ & $\begin{array}{l}\text { Profesor universitario } \\
\text { Perfil } \\
\text { Competencias } \\
\text { Bibliotecologia } \\
\text { Archivística } \\
\text { Fundamentación teórica } \\
\text { Caracterización de la } \\
\text { infornación } \\
\text { Educación bibliotecológica } \\
\text { Enseñanza de la } \\
\text { Bibliotecologia } \\
\text { Teóricos de la Bibliotecologia } \\
\text { Historia de la Bibliotecologia } \\
\text { Epistemologia de la } \\
\text { Bibliotecología } \\
\text { Tipologia de bibliotecas } \\
\text { Teoria de la lectura } \\
\text { Promoción de lectura } \\
\text { Usuarios }\end{array}$ & $\begin{array}{l}\text { College profesor } \\
\text { Profile } \\
\text { Competencies } \\
\text { Library Science / Information Science } \\
\text { Archival Science / } \\
\text { Theoretical basis / Foundations Characterization of the } \\
\text { information } \\
\text { Library Science / Librarianship education } \\
\text { Teaching librarianship } \\
\text { Librarianship theoreticians } \\
\text { Library science history } \\
\text { Library science epistemology } \\
\text { Libraries typology } \\
\text { Reading theory } \\
\text { Reading promotion } \\
\text { Information users }\end{array}$ \\
\hline $\begin{array}{l}\text { Organización y } \\
\text { representación de la } \\
\text { información y el } \\
\text { conocimiento }\end{array}$ & $\begin{array}{l}\text { Organización de la } \\
\text { información } \\
\text { Tratamiento de la } \\
\text { información } \\
\text { Gestión de la información } \\
\text { Lenguajes documentales } \\
\text { Sistemas de clasificación del } \\
\text { conocimiento } \\
\text { Descripción bibliográfica } \\
\text { Indización }\end{array}$ & $\begin{array}{l}\text { Organization of the information } \\
\text { Information processing } \\
\text { Information management } \\
\text { Indexing languages } \\
\text { Knowledge classification systems } \\
\text { Bibliographic description } \\
\text { RDA } \\
\text { FRBR } \\
\text { Indexing }\end{array}$ \\
\hline Gerencia & $\begin{array}{l}\text { Gerencia } \\
\text { Gestión } \\
\text { Administración } \\
\text { Mercadeo } \\
\text { Servicios de información }\end{array}$ & $\begin{array}{l}\text { Management } \\
\text { Administration } \\
\text { Marketing } \\
\text { Information services }\end{array}$ \\
\hline TIC & $\begin{array}{l}\text { Tecnologías de la } \\
\text { información y la } \\
\text { comunicación } \\
\text { TIC } \\
\text { Tecnologia }\end{array}$ & $\begin{array}{l}\text { Information and communications technology } \\
\text { ICT } \\
\text { Technology }\end{array}$ \\
\hline
\end{tabular}




\section{Anexo 2: Estudios en el área de fundamentación teórica}

\begin{tabular}{|c|c|}
\hline Autor, país \& año & Estudios \\
\hline \multirow{2}{*}{$\begin{array}{l}\text { Alfaro López, Héctor. } \\
\text { México, } 2010 . \\
\text { México, } 2011 .\end{array}$} & Estudios epistemológicos de Bibliotecologia. \\
\hline & Hacia la recuperación de la historia en Bibliotecologia y Documentación. \\
\hline $\begin{array}{l}\text { Angelozzi, Silvina. } \\
\text { Argentina, } 2011 .\end{array}$ & Competencias del bibliotecario referencista en el siglo XXI. \\
\hline $\begin{array}{l}\text { Avila Araujo, Carlos Alberto, Brasil, } \\
2014\end{array}$ & $\begin{array}{l}\text { Fundamentos da Ciência da Informação: Correntes teóricas e o conceito de } \\
\text { informação. }\end{array}$ \\
\hline $\begin{array}{l}\text { Castro Escamilla, Minerva. Peni, } \\
2009 .\end{array}$ & La complejidad de la Bibliotecologia: teoria o práctica. \\
\hline $\begin{array}{l}\text { Del Castillo Guevara, Jorge \& Leal } \\
\text { Labrada, Osvaldo. Cuba, } 2006 .\end{array}$ & Bibliotecología: ¿ciencia o técrica? Hacia un nuevo debate. \\
\hline $\begin{array}{l}\text { Escalona Rios, Lina \& Fernández } \\
\text { Cruz, Elba. México, } 2007 .\end{array}$ & Los estudios de egresados del área bibliotecológica: de la teoria a la práctica. \\
\hline $\begin{array}{l}\text { Estrada Cuzcano, Alonso \& Alfaro } \\
\text { Mendives, Karen Lizeth. } \\
\text { México, } 2013 .\end{array}$ & $\begin{array}{l}\text { El método de casos como alternativa pedagógica para la enseñanza de la } \\
\text { Bibliotecologia y las Ciencias de la Información. }\end{array}$ \\
\hline \multirow{2}{*}{$\begin{array}{l}\text { Gutiérrez Chiñas, Agustin. } \\
\text { México, } 2008 . \\
\text { México, } 2005 .\end{array}$} & Identidad profesional de la Bibliotecologia en México a través de su enseñanza. \\
\hline & $\begin{array}{l}\text { La indisciplina en la enseñanza de la disciplina bibliotecológica en México a } \\
\text { nivel licenciatura: una aproximación. }\end{array}$ \\
\hline $\begin{array}{l}\text { Linares Columbié, Radamés \& } \\
\text { Cabrales Hemández, Guzmán. } \\
\text { Cuba, } 2005 .\end{array}$ & Origen y formación de la Ciencia de la Información (1895-1962). \\
\hline $\begin{array}{l}\text { González de Gómez, Maria Nélida. } \\
\text { Brasil, } 2009 .\end{array}$ & $\begin{array}{l}\text { Desafios contemporâneos da ciência da informação: As questőes éticas da } \\
\text { informação. }\end{array}$ \\
\hline $\begin{array}{l}\text { Meneses Tello, Felipe. } \\
\text { Argentina, } 2010 \text {. }\end{array}$ & $\begin{array}{l}\text { La educación bibliotecológica: tema de estudio en el pensamiento de Judith } \\
\text { Licea. }\end{array}$ \\
\hline $\begin{array}{l}\text { Naranjo Vélez, Edilma. } \\
\text { Colombia, } 2005 \text {. }\end{array}$ & $\begin{array}{l}\text { ¿Debe tener bases pedagógicas el bibliotecólogo dedicado a la promoción de la } \\
\text { lectura? }\end{array}$ \\
\hline \multirow{3}{*}{$\begin{array}{l}\text { Parada, Alejandro. } \\
\text { Argentina, 2015. } \\
\text { Argentina, 2013. } \\
\text { Argentina, } 2012 \text {. }\end{array}$} & $\begin{array}{l}\text { Hacia un inventario provisional de las tendencias en Bibliotecologia y Ciencia } \\
\text { de la Información. }\end{array}$ \\
\hline & Perspectivas imaginadas en la Bibliotecologia y Ciencia de la Información. \\
\hline & Bibliotecologia e historia del libro y de las bibliotecas. \\
\hline $\begin{array}{l}\text { Pefla Vera, Tania. } \\
\text { Colombia, } 2008 .\end{array}$ & $\begin{array}{l}\text { Análisis de algunos campos esenciales de la ciencia de la información desde } \\
\text { tres enfoques epistemológicos. }\end{array}$ \\
\hline $\begin{array}{l}\text { Quintanal, Ania. } \\
\text { Cuba, 2007. }\end{array}$ & $\begin{array}{l}\text { Paradigmas dominantes y emergentes en la Bibliotecologia y la Ciencia de la } \\
\text { Información: continuidad y ruptura de la dinámica informacional. }\end{array}$ \\
\hline $\begin{array}{l}\text { Quintero, Nathalia. } \\
\text { Colombia, } 2007 .\end{array}$ & $\begin{array}{l}\text { Aproximación a la epistemología de la bibliotecología como estudio regional } \\
\text { del conocimiento. }\end{array}$ \\
\hline \multirow{2}{*}{$\begin{array}{l}\text { Rendón Rojas, Miguel Ángel. } \\
\text { México, } 2005 \text {. } \\
\text { México, } 2008 .\end{array}$} & Bases teóricas y filosóficas de la bibliotecología. \\
\hline & $\begin{array}{l}\text { Ciencia bibliotecológica y de la información en el contexto de las ciencias } \\
\text { sociales y humanas. Epistemologia, metodologia e interdisciplina. }\end{array}$ \\
\hline \multirow{2}{*}{$\begin{array}{l}\text { Ríos Ortega, Jaime. } \\
\text { México, 2007. } \\
\text { México, 2008. }\end{array}$} & $\begin{array}{l}\text { La teoria en la educación bibliotecológica: directrices básicas para su } \\
\text { enseñanza. }\end{array}$ \\
\hline & $\begin{array}{l}\text { Didáctica de la Bibliotecología: teoría y principios desde la enseñanza de la } \\
\text { ciencia. }\end{array}$ \\
\hline $\begin{array}{l}\text { Setién Quesada, Emilio. } \\
\text { Cuba, } 2007 .\end{array}$ & $\begin{array}{l}\text { Consideraciones sobre la Bibliologia desde la perspectiva de la teoria } \\
\text { bibliológico-informativa. }\end{array}$ \\
\hline
\end{tabular}




\section{Anexo 3: Estudios en el área de OTI}

\begin{tabular}{|c|c|}
\hline Autor, país \& año & $\begin{array}{l}\text { Estudio } \\
\end{array}$ \\
\hline $\begin{array}{l}\text { Bertolini, Maria Violeta \& otros. } \\
\text { Argentina, } 2009 .\end{array}$ & $\begin{array}{l}\text { Nuevos recursos para el tratamiento de la información y la formación de } \\
\text { catalogadores en el siglo XXI. }\end{array}$ \\
\hline $\begin{array}{l}\text { Caldera Serrano, Jorge. } \\
\text { México, } 2015 .\end{array}$ & $\begin{array}{l}\text { Production Research: el nuevo rol del profesional para nuevos tiempos en la } \\
\text { gestión de la información audiovisual. }\end{array}$ \\
\hline $\begin{array}{l}\text { Castro Viguera, Yenifer \& Chávez } \\
\text { Montejo, Yarelys. } \\
\text { Cuba, } 2012 \text {. }\end{array}$ & $\begin{array}{l}\text { La interdisciplinariedad en la formación profesional del analista de información: } \\
\text { propuesta de competencias. }\end{array}$ \\
\hline $\begin{array}{l}\text { Durrieu, Maria Luisa \& otros. } \\
\text { Argentina, } 2011 .\end{array}$ & $\begin{array}{l}\text { Competencias profesionales: una mirada desde la formación en los procesos } \\
\text { técnicos. }\end{array}$ \\
\hline $\begin{array}{l}\text { Garcia, Franco Javier. } \\
\text { España, } 2007 .\end{array}$ & $\begin{array}{l}\text { Ontologias y organización del conocimiento: retos y oportunidades para el } \\
\text { profesional de la información. }\end{array}$ \\
\hline $\begin{array}{l}\text { Garrido Yáñes, Orlanda Angélica. } \\
\text { México, 2007. }\end{array}$ & $\begin{array}{l}\text { Consideraciones del docente en la materia de Catalogación y Clasificación que } \\
\text { se imparte en el Colegio de Bibliotecologia de la Facultad de Filosofia y Letras } \\
\text { de la UNAM. }\end{array}$ \\
\hline $\begin{array}{l}\text { López Zamorano, Cristina. } \\
\text { México, } 2009 .\end{array}$ & El papel del catalogador como gestor de la información. \\
\hline $\begin{array}{l}\text { Martínez Avila, Daniel, San Segundo, } \\
\text { Rosa \& Zurian, Francisco. España, } \\
\text { 2014. }\end{array}$ & $\begin{array}{l}\text { Retos y oportunidades en organización del conocimiento en la intersección con } \\
\text { las tecnologias de la información. }\end{array}$ \\
\hline $\begin{array}{l}\text { Olaran Múgica, María. } \\
\text { España, 2011. }\end{array}$ & Nuevas tendencias en la catalogación a comienzos del siglo XXI. \\
\hline $\begin{array}{l}\text { Picco, Paola. } \\
\text { Brasil, } 2009 .\end{array}$ & $\begin{array}{l}\text { El objeto de la catalogación en el marco de las FRBR y el nuevo código de } \\
\text { catalogación. }\end{array}$ \\
\hline $\begin{array}{l}\text { Ríos Hilario, Ana Belén \& Sánchez } \\
\text { Santos, Maria. } \\
\text { Espaî́a, } 2011 .\end{array}$ & $\begin{array}{l}\text { El perfil actual del personal catalogador: adecuación entre la formación } \\
\text { universitaria y la demanda laboral. }\end{array}$ \\
\hline \multirow{3}{*}{$\begin{array}{l}\text { Rodríguez García, Ariel A. } \\
\text { México, } 2012 \text {. } \\
\text { México, } 2011 \text {. } \\
\text { México, } 2010 \text {. }\end{array}$} & $\begin{array}{l}\text { Claves para la implementación de los lineamientos recursos, descripción y } \\
\text { acceso. }\end{array}$ \\
\hline & Los estudios de caso en la catalogación: sus contextos teórico-prácticos. \\
\hline & El proceso de catalogación: esquemas, principios y prácticas contemporáneas. \\
\hline $\begin{array}{l}\text { Sánchez Garcia, Maria José. España, } \\
2008 .\end{array}$ & La gestión del conocimiento como estrategia de trabajo del documentalista. \\
\hline \multirow{3}{*}{$\begin{array}{l}\text { Santa Anna, Jorge. } \\
\text { Brasil, 2014. } \\
\text { Brasil, 2015. } \\
\text { Brasil, } 2015 .\end{array}$} & $\begin{array}{l}\text { Ampliando o campo de atuaçåo bibliotecária: o bibliotecário como consultor } \\
\text { informacional. }\end{array}$ \\
\hline & $\begin{array}{l}\text { O bibliotecário em face das transformações sociais: De guardiăo a um } \\
\text { profissional desinstitucionalizado. }\end{array}$ \\
\hline & $\begin{array}{l}\text { Desafios para a gestão de estoques de informaçăo frente às coleçổes em } \\
\text { diferentes contextos }\end{array}$ \\
\hline $\begin{array}{l}\text { Sequeira, Deyanira. } \\
\text { Costa Rica, } 2012 .\end{array}$ & Nuevas competencias para gestionar los datos, la información y el conocimiento. \\
\hline $\begin{array}{l}\text { Vargas Rodriguez, Catalina \& } \\
\text { Zamorano Pérez, Angelica. } \\
\text { Chile, } 2012 \text {. }\end{array}$ & La RDA ¿mito o realidad?: panorámica de la catalogación en Chile. \\
\hline
\end{tabular}




\section{Anexo 4: Estudios en el área de administración-gerencia}

\begin{tabular}{|c|c|}
\hline Autor, Lugar \& Año & Estudio \\
\hline $\begin{array}{l}\text { Da Silva, Eduardo Valadares } \\
\text { \& otros. } \\
\text { Brasil, 2008. }\end{array}$ & Perfil dos gestores de informação para a industria capixaba. \\
\hline $\begin{array}{l}\text { Flores de la Fuente, Maria } \\
\text { Lourdes. } \\
\text { Costa Rica, } 2013 .\end{array}$ & Competitividad y liderazgo: elementos vitales para gerentes de unidades de información. \\
\hline $\begin{array}{l}\text { Maspons, Ramon \& Mónica } \\
\text { Montero. } \\
\text { Espaina, } 2010\end{array}$ & El perfil profesional del gestor de la información: information manager. \\
\hline $\begin{array}{l}\text { Téllez Tolosa, Luis Roberto. } \\
\text { Colombia, } 2005 .\end{array}$ & $\begin{array}{l}\text { Pensamiento estratégico y desarrollo de competencias gerenciales: una perspectiva desde } \\
\text { las unidades de información. }\end{array}$ \\
\hline $\begin{array}{l}\text { Varela, Marcela. } \\
\text { Argentina, } 2012 \text {. }\end{array}$ & $\begin{array}{l}\text { Enseñanza innovadora en archivologia para la nueva generación de profesionales } \\
\text { dinámicos en la gestión de la información. }\end{array}$ \\
\hline $\begin{array}{l}\text { Zamora Guzmán, Katia. } \\
\text { Costa Rica, } 2014\end{array}$ & Diagnóstico sobre las competencias gerenciales de los archivistas costarricenses. \\
\hline
\end{tabular}

\section{Anexo 5: Estudios en el área de TIC}

\begin{tabular}{|c|c|}
\hline Autor, país \& año & Estudio \\
\hline $\begin{array}{l}\text { Fuentes de Iturbe, Patricia. } \\
\text { México, } 2006 \text {. }\end{array}$ & Competencias y uso de tecnologias en sistemas de información. \\
\hline $\begin{array}{l}\text { Garduño Vera, Roberto. } \\
\text { México, } 2007 \text {. }\end{array}$ & $\begin{array}{l}\text { Caracterización del docente en la educación virtual: consideraciones para la } \\
\text { Bibliotecologia. }\end{array}$ \\
\hline $\begin{array}{l}\text { Machin Mastromatteo, Juan. } \\
\text { México, } 2012 \text {. }\end{array}$ & Aproximación a las nuevas competencias del bibliotecario. \\
\hline $\begin{array}{l}\text { Morato, Jorge; Sánchez Cuadrado, } \\
\text { Sonia; Fernández Bajón, Maria. } \\
\text { Espaîa, } 2016 .\end{array}$ & Tendencias en el perfil tecnológico del profesional de la información. \\
\hline $\begin{array}{l}\text { Pirela Morrillo, Johann. } \\
\text { Venezuelo, } 2012 \text {. }\end{array}$ & $\begin{array}{l}\text { La formación bibliotecológica para la apropiación sociocrítica de las Tecnologias de } \\
\text { la Información y la Comunicación. }\end{array}$ \\
\hline $\begin{array}{l}\text { Sequeira, Deyanira. } \\
\text { Costa Rica, } 2012 \text {. }\end{array}$ & Nuevas competencias para gestionar los datos, la información y el conocimiento. \\
\hline $\begin{array}{l}\text { Tejada Artigas, Carlos. } \\
\text { España, } 2011 .\end{array}$ & $\begin{array}{l}\text { Competencias tecnológicas de los profesores universitarios en información y } \\
\text { documentación. }\end{array}$ \\
\hline $\begin{array}{l}\text { Uribe Tirado, Alejandro \& } \\
\text { Castaño Muñoz, Wilson. } \\
\text { Colombia, 2010. }\end{array}$ & $\begin{array}{l}\text { La formación en competencias informáticas e informacionales desde la Escuela } \\
\text { Interamericana de Bibliotecologia con el apoyo de una plataforma de E-Learning. } \\
\text { Experiencias y resultados. }\end{array}$ \\
\hline $\begin{array}{l}\text { Uribe Tirado, Alejandro. } \\
\text { Colombia, } 2007 .\end{array}$ & $\begin{array}{l}\text { Los bibliotecólogos colombianos y la adquisición de competencias. Enfasis y } \\
\text { tendencias en la actual formación en tecnologias de la información y la } \\
\text { comunicación. }\end{array}$ \\
\hline
\end{tabular}

